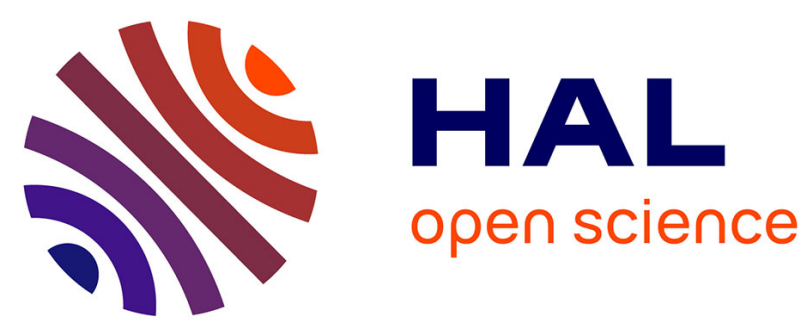

\title{
Les imaginaires de la mobilité. De possibles ressorts pour la mise en durabilité des espaces périurbains?
}

\author{
Benoît Feildel, Hélène Bailleul, Georges-Henry Laffont
}

\section{To cite this version:}

Benoît Feildel, Hélène Bailleul, Georges-Henry Laffont. Les imaginaires de la mobilité. De possibles ressorts pour la mise en durabilité des espaces périurbains?. RTS - Recherche Transports Sécurité, 2014, Les sens des circulations, 2014 (02-03), pp.143-160. 10.4074/S0761898014002064 . halshs01084387

\section{HAL Id: halshs-01084387 https://shs.hal.science/halshs-01084387}

Submitted on 14 Mar 2018

HAL is a multi-disciplinary open access archive for the deposit and dissemination of scientific research documents, whether they are published or not. The documents may come from teaching and research institutions in France or abroad, or from public or private research centers.
L'archive ouverte pluridisciplinaire HAL, est destinée au dépôt et à la diffusion de documents scientifiques de niveau recherche, publiés ou non, émanant des établissements d'enseignement et de recherche français ou étrangers, des laboratoires publics ou privés. 


\section{Les imaginaires de la mobilité. De possibles ressorts pour la mise en durabilité des espaces périurbains ?}

\section{Imaginations of mobility. A possible key for more sustainable periurban areas?}

\section{Benoît Feildel · Hélène Bailleul · Georges-Henry Laffont}

Reçu le 11 novembre 2013 ; accepté le 27 mai 2014

(C) IFSTTAR et Éditions NecPlus 2014

Résumé L'imaginaire géographique est au cœur des rapports que les sociétés entretiennent avec leurs territoires. Ainsi, on connaît l'importance du fruit de l'imagination, la construction mythologique, et son influence sur les représentations et les pratiques de l'espace. On sait notamment le rôle de l'imaginaire qui confère à l'espace sa signification, dans l'extension - aujourd'hui jugée problématique - des périphéries urbaines. Les «mythologies pavillonnaires », la ville à la campagne, le « tous propriétaires », ont énormément œuvré, et ce depuis plusieurs décennies, pour rendre possible le développement de ces nouveaux territoires. Cependant, quand jusqu'à présent l'analyse des imaginaires spatiaux s'est principalement attachée aux espaces de résidence, il demeure selon nous un point aveugle, celui des imaginaires de la mobilité dans les périphéries urbaines - notamment lorsqu'ils sont confrontés aux injonctions sociétales à la durabilité. Partant d'une enquête réalisée auprès d'une trentaine d'habitants des espaces périurbains d'une ville moyenne (Tours), nous proposons d'explorer la place de l'imaginaire lié à la mobilité dans les discours des habitants du périurbain. Nous montrons ainsi, à côté de la mythologie résidentielle, la place qu'occupe l'imaginaire de la mobilité et comment celui-ci ménage des espaces de conciliation compatibles avec les enjeux de durabilité.

Benoît Feildel $(\bowtie)$

École Polytechnique de l'Université de Tours, UMR 7324 CITERES, 35 allée Ferdinand de Lesseps, 37200 Tours

e-mail : benoit.feildel@univ-tours.fr

Hélène Bailleul $(\triangle)$

Université Rennes 2, UMR 6590 ESO, Place du Recteur Le Moal, CS 24307, 35043 Rennes cedex

e-mail : helene.bailleul@univ-rennes2.fr

Georges-Henry Laffont $(\varangle)$

École Polytechnique de l'Université de Tours, UMR 7324 CITERES,

35 allée Ferdinand de Lesseps, 37200 Tours

e-mail : georges-henry.laffont@univ-tours.fr
Mots clés imaginaire $\cdot$ mobilité · périurbain · durabilité

Summary Geographical imagination is deeply rooted in the relations our societies sustain with space. The significance of imagination and mythological constructions of territories is commonly identified as part of our representations and practices. The role of imagination, and its unrealistic function, in the meaning of places is well known, even more in the case of urban peripheries yet identified as problematic. Since decades, suburban mythology, relying classically on the figure of "city to countryside" and the attractiveness of property, has made the production of these new territories possible. But, when the analysis of this myth until now has nearly exclusively concentrated on dwelling places, we propose to reveal a blind spot of imaginations: mobility in suburban areas, especially when considering the sustainability injunction. Based on a qualitative survey carried out in suburban area of a medium size town (Tours, France), this paper renders the role of imaginations towards mobility in the today life of inhabitants. We show how mobility mythology, as well as dwelling myth, influences suburban way of life. Moreover, we insist on the capability of these imaginations to offer spaces of conciliation encouraging more sustainable way of life.

Keywords imagination - mobility - suburban area . sustainability

\section{Introduction}

La question des imaginaires en lien avec l'espace n'est pas nouvelle en soi [1]. En revanche, elle revêt une dimension singulière et riche lorsqu'il s'agit de saisir les ressorts et les mécanismes sous-jacents à la réalisation des formes d'occupation de l'espace, en particulier périurbaines [2]. En effet, depuis les travaux fondateurs menés sur la préférence massive des Français pour l'habitat pavillonnaire [3], la 
compréhension des imaginaires attachés au logement, dans un premier temps, a permis d'éclairer ce qui, pour un certain nombre de contempteurs, passait pour un rêve déraisonnable et qui pourtant depuis lors n'a eu de cesse de se concrétiser [4]. L'imaginaire géographique présente ainsi une clé de lecture qui s'avère particulièrement pertinente pour comprendre à travers son actualité, et les modalités de son articulation avec le réel, les relations qu'entretiennent les individus et les groupes avec les espaces, notamment avec ces espaces périurbains, «manifestement peu denses et réputés relativement homogènes, situés en discontinuité paysagère par rapport aux agglomérations urbaines » [5].

Loin de s'opposer au réel, l'imaginaire - géographique en particulier - y a partie liée et ce, de multiples façons. Mythe, imaginaire et représentation constituent un ensemble de notions étroitement liées, qu'il convient de mieux saisir pour comprendre la nature même de cette relation au réel. Le mythe est un récit portant généralement sur les origines d'une civilisation, de l'homme, sur ses fins, sur sa nature ou encore son inscription dans la société. En tant que tel, le mythe non seulement peut pousser à agir ; il est alors mobilisateur, mais il peut aussi indiquer comment agir et, en ce sens, avoir valeur de norme. Ainsi, le mythe s'impose, partiellement mais fortement, à l'individu. À la différence du mythe, pour lequel les dimensions de norme et de morale sociale dominent, l'imaginaire, lui, se caractérise par une forte dimension affective. Il y a, dans l'imaginaire, de l'envie ou de la peur. L'imaginaire, non seulement, a du sens mais donne également du sens à ce qu'il ne contient pas [6,7]. Là où le mythe est une construction collective, l'imaginaire, lui, peut être individuel. Des imaginaires peuvent coexister, s'opposer, se composer. Quant à la représentation, elle serait l'unité de base des imaginaires et des mythes, sans morale, ni envie. Elle porte sur des objets ou des situations et à ce titre fait le lien avec le réel, permet à l'individu de lui donner sens [8]. Premier niveau de signification, la représentation, à la fois individuelle dans son procès cognitif, et collective par la capacité à être partagée, intériorisée, transmise par la culture, est ce qui va être mobilisé dans les imaginaires et consolidé dans les mythes. Dès lors, représentations, imaginaires et mythes doivent être considérés comme différents niveaux permettant d'atteindre la manière dont les individus mettent en significations leurs univers géographiques [9].

Si la prégnance et le poids des imaginaires associés à la maison individuelle ont été montrés depuis plusieurs décennies déjà, notre propos ici sera de partir à la recherche d'autres constructions signifiantes, que l'on reconnaît aujourd'hui encore comme participant pleinement du phénomène de périurbanisation. On reviendra, dans une première partie, sur cet objet fondateur - et encore largement fécond - de l'imaginaire périurbain, mais notre propos s'attachera principalement à éclairer un autre versant, qui nous semble encore relativement peu exploré : la fonction imaginaire qui s'attache aux mobilités. On supposera de cet autre versant qu'il est tout aussi important, en particulier dans les espaces périurbains, et dès lors on avancera l'hypothèse que le développement périurbain n'est pas seulement le produit d'un imaginaire que les individus et les sociétés associent à l'espace résidentiel. Sa concrétisation, en particulier dans le cas français qui nous intéressera ici, serait non seulement le résultat de la conjonction de plusieurs facteurs, socio-économiques avant tout, mais dont l'une des circonstances, conjointement à l'avènement d'une ère de la mobilité [10], serait la production d'un imaginaire également propre à cette condition mobilitaire. On insistera également sur la capacité de cet imaginaire à être remodelé à l'aune de la durabilité et des discours ambiants sur le périurbain.

Le mouvement de périurbanisation caractéristique, en premier lieu, d'un phénomène de dissociation des lieux d'emploi et de résidence, s'accomplit et s'accroît dès lors que la mobilité, à travers notamment ses progrès techniques, permet un affranchissement croissant vis-à-vis des contraintes de l'espace et du temps [11]. Pour autant, comme le suggère Dodier [12], la prise en compte des pratiques spatiales des ménages périurbains, et en particulier leurs manières de se déplacer, relativement à l'occupation et à l'appropriation de l'espace du logement, n'a finalement émergée que récemment dans le paysage scientifique. Nonobstant les travaux précurseurs de Piolle [13], il aura fallu attendre le début des années 2000 pour que l'étude du sens des mobilités ne devienne véritablement déterminante [14,15]. Ainsi, la question des mobilités quotidiennes n'est que récemment interrogée pour les qualités qui s'y rapportent ; c'est-à-dire au-delà de la question de ses quantités et de ses modalités, pour ses significations et ses valeurs $[16,17,18]$. Dans cet article, notre but sera donc de participer à la compréhension des imaginaires, des mythes et des représentations, associés à la mobilité dans les espaces périurbains. Comment se construisent-ils ? Quels en sont les ressorts ? Comment s'articulent-ils avec les imaginaires spatiaux et sociaux propres aux périphéries urbaines ? Comment participent-ils de l'institution de la réalité territoriale périurbaine?

Pour cela, nous nous appuyons sur les données empiriques collectées dans le cadre d'une enquête ${ }^{1}$ menée auprès d'une trentaine d'habitants des espaces périurbains de l'agglomération de Tours [19]. Grâce à cette enquête, réalisée entre septembre 2010 et juin 2011, qui cherchait

\footnotetext{
${ }^{1}$ Cette enquête a été réalisée dans le cadre de la recherche « Le périurbain à l'épreuve des modèles d'habiter. La viabilité périurbaine entre théorie(s) et pratique(s) » [17] financée par le Plan urbanisme construction architecture (Puca); ministère de l'Écologie, du Développement durable, des Transports et du Logement.
} 
principalement à amener les enquêtés à évaluer leurs modes d'habiter périurbains en confrontant la réalité de leurs pratiques géographiques avec les discours ambiants porteurs d'une injonction à la durabilité, nous proposons à la fois de restituer une partie de cet imaginaire mobilitaire et d'en cerner les évolutions récentes. Comment l'imaginaire de la mobilité est-il traversé par les valeurs sociétales de durabilité ? Quelles sont les conséquences de l'injonction à la durabilité ? Comment participe-t-elle à renouveler ces imaginaires ? Et dans quelle mesure ces renouvellements influent-ils ou pourraient-ils potentiellement influer sur l'institution d'une nouvelle réalité périurbaine ? Après un bref rappel des mythologies qui ont historiquement peuplé les imaginaires des périphéries urbaines, puis une présentation de la méthode mise en œuvre pour atteindre le sens des mobilités quotidiennes dans ces espaces périphériques, nous illustrons les mutations à l'œuvre dans l'imaginaire périurbain contemporain en montrant l'influence de la norme de durabilité et la façon dont elle participe, dans une certaine mesure, au renouvellement des imaginaires de la mobilité.

\section{Les mondes imaginaires des périurbains}

\subsection{L'idéal de la maison individuelle au plus près de la nature}

Le périurbain repose sur une mythologie riche. Si notre propos n'est pas de décomposer élément par élément les raisons et motivations ayant conduit à cette «passion pavillonnaire » [4], néanmoins on s'attachera ici à décrypter quelques caractéristiques et dimensions de ce périurbain, qui cristallise bon nombre d'images, à commencer par le pavillon. «L'idéalisation de la maison individuelle au plus près de la nature découle d'une longue histoire, que l'on renvoie communément en occident au mythe arcadien » [20]. Ce propos met en lumière au moins deux imaginaires fondateurs : celui de la maison individuelle, et étroitement lié à ce premier, au point presque de s'y confondre, celui de la nature. Qu'elle soit chalet, longère, mas ou simple pavillon fabriqué en série, la maison fonde une relation à un territoire, à la famille, à l'histoire et à la société [21]. Elle permet de s'ancrer et sa possession apparaît comme une garantie d'avenir pour soi comme pour ses enfants. L'imaginaire de l'habitat individuel tient donc en grande partie dans l'inscription individuelle, dans une filiation, dont chacun est conscient et qui le dépasse en même temps. Dans sa finalité première, la maison symbolise l'expression de la quête du lieu originel - naturel par définition nécessairement perdu et par conséquent fantasmé. Par extension, le périurbain - lieu d'expression privilégiée de cette forme d'établissement humain - symbolise un espace du possible déploiement d'une communauté villageoise retrouvée et elle aussi fantasmée. D'autres éléments encore entrent dans la composition complexe de cet imaginaire. « La maison constitue un capital à plusieurs titres : culturel, social, économique. C'est un investissement qui cristallise désirs, raison, représentations ainsi que normes attachées à l'idée de réussite sociale et individuelle » [22]. Le désir d'être propriétaire " explique une grande part de cette aspiration à vivre en pavillon dans le périurbain » [12]. La maison s'impose comme la double traduction d'un investissement monétaire et temporel sur le long terme. « Être propriétaire d'une maison individuelle est également vécu comme un élément du processus de valorisation sociale »[23]. Ces aspects, qui n'occultent en rien l'importance de la question de la rente foncière et de la difficulté à accéder à un bien immobilier plus proche du centre urbain, sont fondamentaux dans la constitution de l'imaginaire pavillonnaire, au même titre que le désir de nature qui systématiquement l'accompagne. L'extraordinaire engouement que le monde occidental connaît pour la maison individuelle et le développement exponentiel, depuis le sortir de la seconde guerre mondiale, des organisations socio-spatiales périurbaines, s'expliquent tous deux, lorsqu'ils se rejoignent, par la collusion entre un imaginaire pastoral, traditionnellement associé au mythe arcadien, et un imaginaire social qui fait de la maison un capital de divers ordres.

Enfin, en tant que catégorie commune, le périurbain apparaît dans la sphère médiatique dans les années 1990 et « il ne s'agit pas seulement, pour les journalistes, de caractériser un phénomène selon les normes de l'écriture scientifique, mais aussi de s'interroger sur sa dimension éthique et de l'ériger en question de société » [24]. Les médias participent alors de la mise en scène d'un espace pertinent, lui attribuant un certain nombre de valeurs. Le succès périurbain réside dans le fait qu'il répond, dans l'imaginaire, à un certain nombre d'attentes comme la quête d'espace, de calme, de sécurité. Ainsi, progressivement un idéal résidentiel, fondé sur la recherche d'un cadre de vie domestique de qualité et susceptible de conférer bien-être et équilibre, succède au seul idéal de la maison. Le périurbain apparaît comme la concrétisation de l'idéalisation de la campagne, ramassée en un cadre de vie idyllique et bucolique et accompagnée du rejet de la ville moderne dense et verticale, ou encore comme la réalisation paradoxale d'une volonté délibérée de se soustraire à l'intensité de la vie urbaine et en même temps de satisfaire un désir de citadinité. L'émergence du périurbain, dans son volet symbolique, est la conséquence d'une tendance à entrevoir le bonheur dans une forme hédoniste et familiale, où les avantages de la ville et de la campagne se trouvent, comme par enchantement, associés, et dont la condition de réalisation, dans l'imaginaire collectif, est la mobilité. 


\subsection{Un mythe oublié?}

\section{L'imaginaire de la mobilité dans les espaces périurbains}

Depuis cinq décennies désormais, le mouvement de desserrement des espaces résidentiels autour des centres urbains n'a pas fléchi, notamment facilité par le développement et l'accessibilité aux mobilités. Ce phénomène, dont les conditions de réalisation sont principalement et avant tout économiques, porte la marque de l'essor et de la généralisation des mobilités. Pour autant, le rôle joué par la mobilité dans la constitution de ces nouveaux territoires, fortement hétérogènes, est demeuré pendant longtemps principalement questionné sous l'angle des quantités de déplacements, de leur rationalisation, même si le sens que lui confèrent les populations habitantes a été investigué depuis quelques années [18]. Alors que l'on assiste aujourd'hui au démontage des imaginaires périurbains et à leur contrepoint urbain, alors qu'est désormais posée la question d'une durabilité possible, d'une urbanité à inventer qui serait propre aux espaces périurbains, qu'en est-il des imaginaires de la mobilité dans les espaces périurbains ? Face au discours scientifico-technique contre les «étalés », suivant l'interpellation que nous adresse Vanier [25], a-t-on tout dit des mobilités et des conditions d'un avenir périurbain ? L'imaginaire que véhiculent ces mobilités ne peut-il pas servir un projet périurbain, réinventant le rapport au territoire?

Avant d'envisager les réponses possibles à ces différentes questions, on commencera par un appel à la prudence qui vient contrebalancer une certaine pensée catastrophiste, et relativiser les idées qui, campant la mobilité dans un imaginaire purement techniciste, n'envisagent ses effets que par l'effacement de la relation aux espaces géographiques, aux territoires. Au contraire, il s'agit bien d'essayer de comprendre les flux et leurs significations, d'élucider comment la mobilité est un vecteur en actes de la construction du sens que les individus donnent au monde qu'ils habitent [15]. L'individu mobile s'approprie des « territoires circulatoires »[12], développe un ensemble de routines mobilitaires autour de trajets, de parcours, de routes, jalonnées d'étapes, de repères, de références. Il s'identifie aux objets de la mobilité, tronçon routier, rame de train, paysages en mouvement, itinéraires, cheminements, etc. Il s'y reconnaît, de même qu'il les partage avec un ensemble d'autres individus, au point de se retrouver autour de ces objets identificatoires et de former un véritable collectif, socialement constitué [26]. Ainsi, loin d'apparaître comme un simple moyen technique désincarné, la mobilité non seulement s'affranchit de la stricte contrainte des réseaux, mais par là même, revêt une dimension signifiante. Surtout, elle se développe, se réalise dans l'actualisation d'un imaginaire qui lui est également propre et dont il convient dès lors de prendre toute la mesure.
Décrire l'imaginaire de la mobilité contemporaine nous permet de poser les bases d'une approche compréhensive des territorialités périurbaines contemporaines. Il nous faut rappeler alors que cet imaginaire apparaît pour certains auteurs « comme une boussole privilégiée pour cerner les transformations de la modernité »[7]. La mobilité se révèle porteuse d'un sens qui lui est à la fois propre et qui, par bien des égards, reflète les évolutions de nos sociétés [9]. D'abord ancrée dans la dimension du voyage, la mobilité est synonyme au départ de liberté, d'une capacité de se détacher d'un ici pour tendre vers un ailleurs ; ailleurs lui-même synonyme d'aventure, de nouveauté, de découverte de soi et de transformations existentielles. La mobilité revêt, ensuite, dans la période contemporaine, un sens beaucoup moins idéalisé, à la mesure de sa généralisation et de sa banalisation. Entrant dans le règne de l'ordinaire et de la banalité, l'imaginaire de la mobilité se heurte de plein fouet à ce qui faisait sa spécificité. La mobilité auparavant extraordinaire et aux consonances de voyage et d'aventure devient un attribut des modes de vie quotidiens. Le culte du mouvement, la mobilité pour la mobilité, indissociable des espaces qui la caractérisent, purement fonctionnels, et ancré dans l'idéologie libérale ; la mobilité qui tend à annihiler toute idée de dehors, de même que toute idée de dedans, et qui renvoie au désir d'un style de vie mondialisé, d'une maîtrise de l'espace et du temps sans borne ni limite ; tout cela forme les totems d'un imaginaire profondément renouvelé de la mobilité, dont la transformation révèle en premier lieu un renversement de la valeur «mobilité » par sa banalité et sa quotidienneté. C'est ainsi qu'à l'aube du XXI siècle, un certain nombre de chercheurs en sciences sociales ont pu dépeindre, en en faisant l'éloge d'abord, puis de façon plus nuancée, les traits caractéristiques d'une « hypermobilité » comme un mode de vie pleinement assumé et dès lors revendiqué [27]. Contre la vision traditionnelle du sédentaire, stable et immuable par nature, dont la qualité résulte d'une certaine permanence dans l'espace et dans le temps, progressivement la mobilité est devenue un nouvel attribut de la puissance, sa dimension existentielle prenant de plus en plus d'importance [7].

Parallèlement à l'avènement d'une société « hypermobile »-concernant dans les faits les catégories de population les plus nanties - on assiste également, comme en réaction, à une critique de plus en plus importante des effets de ce mouvement frénétique. La mobilité elle-même est désormais l'objet d'un ensemble de griefs de plus en plus nombreux, participant d'une forme d'atomisation de ses imaginaires. C'est ainsi qu'à côté de valeurs traditionnelles - la quête de l'exotique d'abord, l'hypermobilité synonyme de puissance ensuite - on voit poindre un renouvellement des imaginaires de la mobilité, sous la forme parfois de véritables contre-cultures [28]. Face à l'accélération de la vie quotidienne, facilitée par la vitesse de déplacement 
des hommes et des informations, de nouveaux imaginaires fondés sur le ralentissement et la (re)valorisation des relations de proximité se font jour, s'incarnant notamment dans le développement d'" une mobilité qui prend son temps »[29]. Dès lors, ce qui formait les totems de la mobilité en temps de modernité est aujourd'hui largement remis en question, avec en premier lieu un refus et une dénonciation de l'accélération de la vie quotidienne, mais aussi, pour partie, de l'éloignement aux êtres et aux biens qui peut y être associé. Cette transformation des attributs positifs de la mobilité révèle alors un renversement de valeur à l'œuvre dans nos sociétés, sous l'effet notamment d'une critique du modèle libéral et de ses effets pervers sur l'environnement.

Dans nos sociétés postmodernes, les valeurs et mythes associés à la mobilité sont désormais caractérisés par ces différents mouvements, lesquels souvent s'opposent en tout point et contribuent ainsi à la production d'imaginaires réversibles, en fonction des pratiques et des représentations de chacun. Dès lors, il s'agit de montrer comment ces imaginaires de la mobilité, atomisés, réversibles ou encore hybrides, s'actualisent dans le cas spécifique des espaces périurbains. Il devient essentiel de comprendre comment ces mouvements de fond, s'exprimant de façon privilégiée dans les imaginaires associés à la mobilité, peuvent avoir un impact sur les pratiques et les représentations quotidiennes. Comment les périurbains font avec ces imaginaires ? Finalement, qu'en est-il de cet imaginaire de la mobilité lorsque l'on s'intéresse spécifiquement aux espaces périurbains, à des espaces dont l'existence même est étroitement dépendante des pratiques de mobilité ? Connaissant le poids et la force des imaginaires spatiaux attachés aux espaces périurbains, qu'en est-il du sens des mobilités dans ces espaces? Qu'en est-il de cet imaginaire face à une injonction au développement durable dont les périurbains sont désormais la cible privilégiée?

\section{Enquêter les imaginaires : \\ la mobilité au prisme de l'injonction à la durabilité}

\subsection{Mythe ou réalité : \\ les effets de l'injonction à la durabilité}

L'impérieuse nécessité de mettre en place les conditions d'une organisation et d'une pratique durables des territoires, et sa déclinaison sur les plans sociaux, économiques et environnementaux, nous amène au constat que le mode d'habiter périurbain se trouve confronté à une injonction à la durabilité qui n'est certainement pas sans conséquences sur les imaginaires géographiques qui s'y rattachent. En effet, depuis les années 1990 et l'avènement d'une nouvelle idéologie sociale fondée sur la durabilité, les textes, articles, recherches n'ont eu de cesse d'accuser ce mode d'habiter de tous les maux [30]. Sans chercher ici à délimiter et à caractériser précisément les modalités que revêt cette injonction à la durabilité, nous pouvons cependant en esquisser certains traits et ainsi montrer dans quelle mesure elle entre potentiellement en relation, voire en conflit, et engendre certaines transformations des imaginaires associés à la mobilité.

L'injonction à la durabilité s'inscrit dans une redéfinition plus large des rapports qu'entretiennent nos sociétés occidentales au risque et à l'incertitude [31]. Ces nouveaux rapports fondent pour partie l'omniprésence et la prédominance à la fois médiatique, politique et opérationnelle, des problématiques de développement durable, au-delà de la nécessité désormais devenue incontournable de mettre en œuvre les conditions d'un développement qui réponde aux besoins du présent sans compromettre la capacité des générations futures à répondre aux leurs. Face à cette nécessité de réaliser des projets et de développer des stratégies afin de maîtriser l'avenir, la question de la durabilité apparaît comme un cadre normatif se déclinant à la fois en programmes et politiques permettant de définir collectivement les objectifs du développement durable, mais aussi sous formes de règles de conduite incitant les individus à développer un ensemble de pratiques plus vertueuses. En matière d'aménagement des territoires, cette quête de la durabilité se traduit en principes et outils de gestion appliqués à différentes échelles territoriales, de façon sectorielle ou intégrée ${ }^{2}$, dans un but toujours affirmé de réduire l'impact des activités humaines sur l'environnement, principalement en contrôlant et limitant le développement urbain.

Les objectifs affichés dans le cadre de ces programmes et politiques se déclinent en orientations et actions fortes : réduire l'usage de l'automobile, notamment en luttant contre l'étalement urbain par une densification raisonnée. Face à l'étalement urbain, en particulier, la notion de « ville compacte » s'affirme et vient ainsi s'opposer à un modèle de «ville étalée » dont le périurbain serait l'archétype même, et ce malgré la très grande diversité des formes et des organisations périurbaines. Pour Jacques Lévy, entre autres, les modèles d'urbanité soutenus par ces différentes conceptions de l'organisation des espaces habités sont à mettre en lien, directement et de façon étroite, avec la question du développement durable et l'injonction à laquelle celui-ci nous oblige. « Les acteurs majoritaires

\footnotetext{
${ }^{2}$ C'est ainsi que les plans climat-énergie territoriaux, apparus dans le cadre du Plan climat national (2004) ou encore les schémas de cohérence territoriale, créés dans le cadre de la loi relative à la Solidarité et au renouvellement urbains (1999), se sont vus récemment renforcés par la loi portant Engagement national pour l'environnement (2010).
} 
- ceux qui continuent de souhaiter un monde urbain structuré par la résidence individuelle et l'automobile, avec toutes les conséquences que cela implique - sont de moins en moins légitimes »[32]. Parce que la densité influerait grandement sur les modes de déplacements et les distances parcourues, parce qu'une plus grande densité serait synonyme d'une moindre consommation de carburants [33], et donc d'émissions de gaz à effet de serre, le modèle de la ville compacte, en agissant directement sur la forme urbaine, vise à réduire l'étendue de la mobilité quotidienne et ses méfaits. Dès lors, il s'agit de limiter la diffusion de l'urbanisation, notamment en favorisant le retournement des mobilités résidentielles. De fait, le mouvement de périurbanisation, assimilé à une production accrue d'espaces artificialisés au détriment des espaces agricoles et naturel, et à un accroissement du nombre de kilomètres parcourus du fait d'un taux de motorisation des ménages ne cessant lui-même d'augmenter, s'impose aujourd'hui comme la cible territoriale privilégiée de l'injonction au développement durable. La tendance actuelle est de qualifier et d'évaluer le périurbain comme «non durable ». Ces critiques impliquent, de façon sous-jacente mais bien réelle, que le périurbain serait responsable des divers maux qui entravent la mise en œuvre d'un développement territorial durable. Cette approche, fortement stigmatisante à l'égard des comportements des périurbains, n'en est pourtant pas moins débattue au sein de la communauté scientifique [34]. Elle nous invite à questionner l'effectivité de la «non durabilité » supposée, en confrontant, par exemple, l'un des principaux vecteurs du mouvement de périurbanisation - les populations périurbaines elles-mêmes - à l'injonction qui leur est faite de tendre vers davantage de durabilité.

À ce mode d'habiter, impliquant la résidence mais aussi la mobilité dans les espaces périurbains, nous avons donc souhaité, par le biais de notre enquête [19], opposer cette injonction à la durabilité. Nous sommes donc partis du principe qu'une enquête sur le rapport à l'espace des habitants du périurbain, englobant le fait de résider, mais aussi et surtout le fait de s'y déplacer, d'y construire une territorialité particulière, pouvait nous renseigner sur les représentations et les mutations de l'imaginaire qui pouvaient naître de cette injonction. La confrontation de l'imaginaire périurbain à l'injonction de durabilité constitue de la sorte une situation qui permet de faire émerger tout ce qui explique, donne de la valeur et enfin (ré)enchante des pratiques quotidiennes de l'ordre du routinier et de l'habitude. C'est en amenant les enquêtés à une réflexivité sur leurs propres pratiques, qu'il a ainsi été possible de faire émerger, de ces imaginaires géographiques, les imaginaires propres à la mobilité périurbaine, et de comprendre au-delà de la stricte dimension fonctionnelle du déplacement, son sens et ses significations potentiellement multiples. Considérant qu'il est tout aussi capital de comprendre et d'analyser de quelle(s) manière(s) les individus définissent eux-mêmes les conditions du durable, au regard notamment de leurs pratiques actuelles, de leur capacité à les faire évoluer, de sorte d'assurer la pérennité de leur mode de vie, nous avons donc cherché à interroger la mutation des imaginaires de la mobilité dans le quotidien périurbain, et sa contribution potentielle à l'établissement d'un périurbain durable.

\subsection{Interroger les imaginaires mobilitaires}

C'est pour comprendre les effets de l'injonction au développement durable sur les pratiques spatiales des habitants des périphéries urbaines, et en particulier sur le sens que revêtent pour eux leurs mobilités, que nous avons mis en œuvre l'enquête Périvia [19]. À travers cette enquête, nous n'avons pas tant cherché à comprendre l'imaginaire attaché aux espaces périurbains, par ailleurs déjà largement exploré, mais le sens particulier que prennent les différentes formes de mobilité chez les habitants. De ce fait, nous avons opté pour une méthode de captation originale [35], nous permettant d'accéder aux mobilités quotidiennes, et de les interroger par l'utilisation combinée de deux outils méthodologiques. Nous avons ainsi mis en œuvre un relevé GPS exhaustif des déplacements des individus, et nous nous sommes appuyés sur la représentation cartographique très précise de ces déplacements pour tendre, lors d'un entretien de réactivation fondé sur ce support graphique, vers le sens que les individus confèrent à leurs mobilités et les représentations, les imaginaires et les mythes que celles-ci véhiculent. C'est donc, en nous adressant directement à l'individu, à l'habitant des périphéries urbaines, en le confrontant à la réalité de ses déplacements, en écoutant sa réaction, et en lui demandant de nous délivrer le sens de sa mobilité spatiale, que nous avons pu atteindre l'imaginaire des mobilités périurbaines.

L'enquête que nous avons menée, à partir d'un terrain d'étude périurbain situé dans une ville moyenne française, l'agglomération de Tours, a ainsi visé à expliciter les liens et les articulations entre les dimensions matérielle et idéelle de la mobilité. Le choix d'une analyse fine des mobilités quotidiennes d'un ensemble d'habitants de ces espaces périurbains devait nous permettre d'apporter des éléments de compréhension sur la structuration des modes d'habiter et les pratiques mobiles de ces habitants. Il s'agissait de comprendre le sens particulier que prennent les différentes formes de mobilités dans la construction du rapport à 
l'espace, autrement dit la manière de faire de ces habitants dans les espaces périurbains, dont l'organisation des activités quotidiennes nécessite jour après jour une mobilité spatiale importante. Enfin, cette ambition compréhensive s'est également articulée avec un enjeu pragmatique de premier plan, celui du ménagement des espaces périurbains pour la réalisation de leur durabilité. Comprendre le sens de mobilités dans les périphéries urbaines, sans sous-estimer l'ensemble des mécanismes économiques qui dictent en grande part les choix de localisation des populations, nous a conduits à interroger les modes d'habiter périurbains, leur capacité à tendre vers une organisation sociale et spatiale compatible avec les enjeux de durabilité et le rôle des imaginaires dans la possibilité d'un tel avènement.

Le protocole mis en œuvre a dès lors consisté à interroger un ensemble d'individus (Tableau 1) identifiés comme périurbains ${ }^{3}$. Ils pouvaient l'être tout d'abord du fait de la localisation de leur résidence principale dans le « géotype » périurbain et de leur lieu de travail dans l'espace urbain. Ils pouvaient l'être aussi - dans un souci de mise en perspective et donc dans une moindre proportion - par le fait d'une localisation résidentielle toujours périurbaine, mais d'un lieu de travail lui-même périurbain, ou encore $\mathrm{du}$ fait d'un lieu de travail périurbain et d'une résidence citadine, nécessitant néanmoins une mobilité quotidienne importante (Tableau 2). L'échantillon ainsi constitué visait autant à questionner l'effet du contexte périurbain sur les mobilités que l'expression d'un savoir-faire avec l'espace, un « habitus mobilitaire » [36] relatif aux individus et aux groupes sociaux, à leurs histoires, leurs habitudes, leurs représentations. Du fait de cet objectif double, et de la volonté de questionner ces différentes dimensions sans les dissocier, le protocole d'enquête a tout d'abord établi la nécessité d'un enregistrement de l'ensemble des déplacements des individus, par une mesure réaliste et objective à l'aide d'un dispositif GPS. La suite du protocole

\footnotetext{
${ }^{3}$ Le nombre relativement faible $(\mathrm{n}=37)$ de personnes enquêtées nous a conduits à favoriser, pour la constitution de l'échantillon, une certaine diversité des profils, en ayant dès lors recours à une méthode de sélection ciblée et non aléatoire. Plutôt qu'une stricte représentativité des populations périurbaines, nous avons cherché, conformément à nos hypothèses, à recruter des individus présentant des situations différenciées, en particulier du point de vue de leur localisation résidentielle. Partant de l'hypothèse que ce critère serait déterminant pour la mobilité, nous avons construit l'échantillon de sorte à couvrir un gradient de situations géographiques, du plus proche au plus éloigné du centre de l'agglomération tourangelle. Ensuite, l'usage de techniques de recrutement variées, par réseau de connaissance et par effet « boule de neige », par recrutement direct d'usagers des transports en commun (TER, cars départementaux) ou encore par prise de contact avec des groupes ou des associations de promotion du développement durable et/ou des transports alternatifs (co-voiturage, vélo), nous a assurés d'une relative diversité des modes de transport et des niveaux d'engagement en matière de développement durable.
}

a dès lors été construite de façon à permettre l'expression des valeurs, des représentations et des imaginaires liés à la mobilité, en intégrant, par le biais d'un entretien de réactivation approfondi, une mise en récit des mobilités aux différentes étapes de la vie. Le protocole d'enquête ainsi établi a bien été construit dans le but, d'une part, de collecter les mouvements observables d'un ensemble d'individus, mais aussi, d'autre part, de comprendre les sens des mobilités à l'échelle individuelle, depuis les conditions exprimées de leur réalisation quotidienne, jusqu'à l'inscription de ce savoir-faire mobile dans une trajectoire biographique.

Dans un premier temps, nous avons équipé 37 individus d'un récepteur GPS. Ce faisant, nous avons pu enregistrer l'ensemble des déplacements effectués par ces individus durant une semaine type, comprenant une série de jours travaillés et non travaillés. À l'issue de cette première étape, nous avons obtenu un relevé systématique de l'ensemble des pratiques spatiales des 37 individus enquêtés, comprenant leurs déplacements mais aussi les temps passés aux différents lieux d'activité (Carte 1).

L'exploitation des informations récoltées grâce à l'équipement GPS, particulièrement riches, car fines et exhaustives, n'a cependant pas prioritairement visé un traitement statistique de la donnée quantitative ainsi produite. Nous nous sommes d'abord intéressés à la représentation cartographique des mobilités ainsi révélées. Avant tout, le protocole d'enquête a été construit de façon à utiliser les traces GPS, sous forme de cartographie interactive et dynamique, comme outil de réactivation susceptible de fonctionner comme un embrayeur de discours [15]. La confrontation de l'individu à son relevé GPS personnel, à la vision objective de ses propres mobilités, a donc été mise en œuvre lors d'un entretien de réactivation. Cet entretien s'est ainsi apparenté à une épreuve de réalité [37] qui visait avant tout à susciter un "discours d'existence » [38], mobilisant la capacité réflexive de l'enquêté, jusqu'à atteindre la représentation qu'il se fait de ses mobilités et l'imaginaire qui leur est attaché. Cet entretien a également eu pour fonction d'appeler à une mise en perspective de la mobilité quotidienne de l'individu, à différentes échelles temporelles. L'enquêté s'est ainsi vu invité à comparer et à évaluer le schéma type de ses mobilités actuelles à l'aune des différents schémas et des habitudes de mobilités qu'il a connu tout au long de sa trajectoire biographique (Figure 1). L'entretien de réactivation, sur la base du relevé objectif par GPS, a pris la forme d'un véritable « récit de mobilités » [35] au cours duquel l'individu a pu rendre explicite le sens de ses mobilités quotidiennes, en faisant appel pour cela à des éléments plus anciens dans son propre parcours de vie.

Au cours de ce deuxième temps, le protocole d'enquête s'est donc orienté vers l'énonciation d'un « récit de 


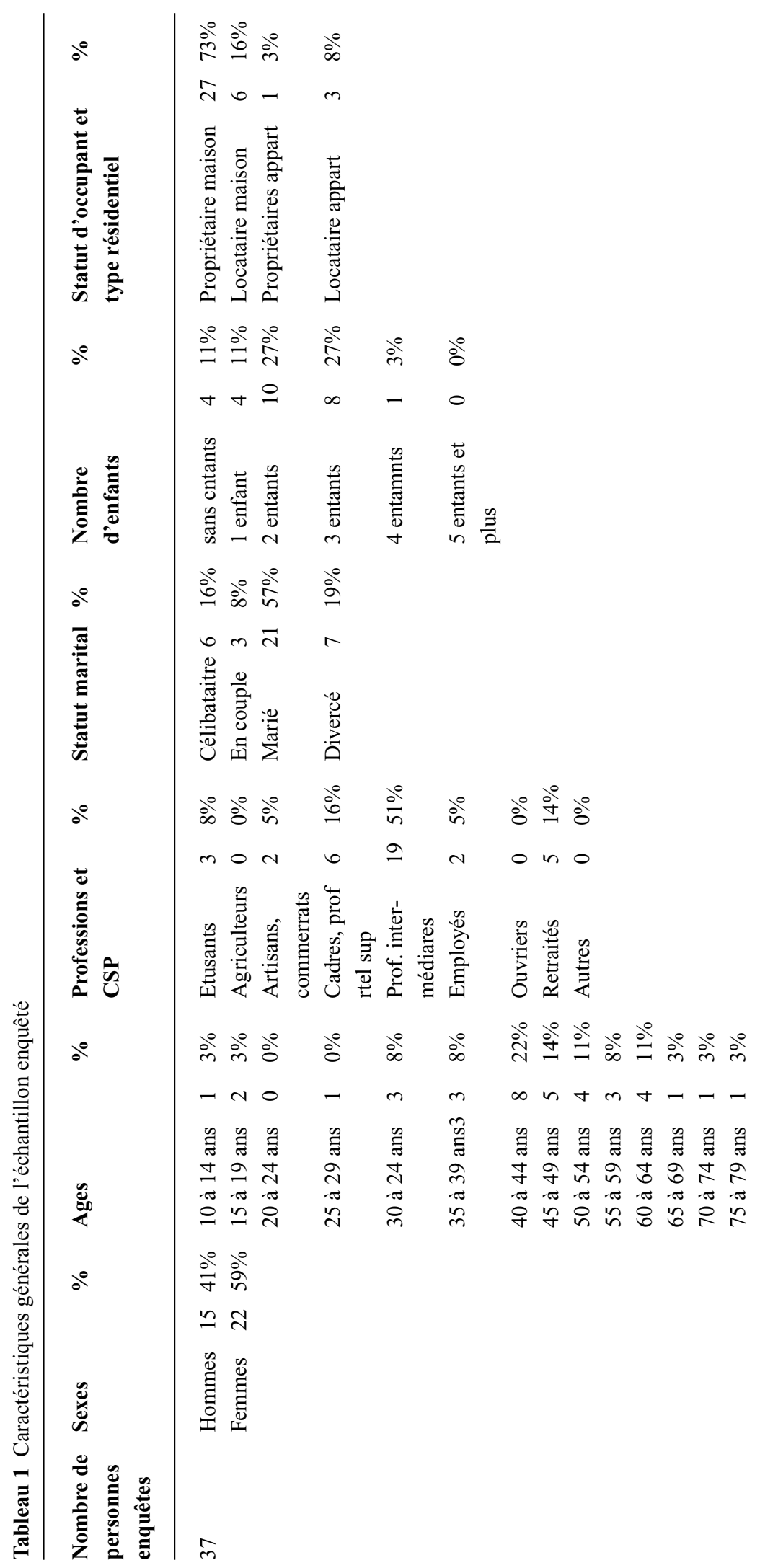

Necplus 


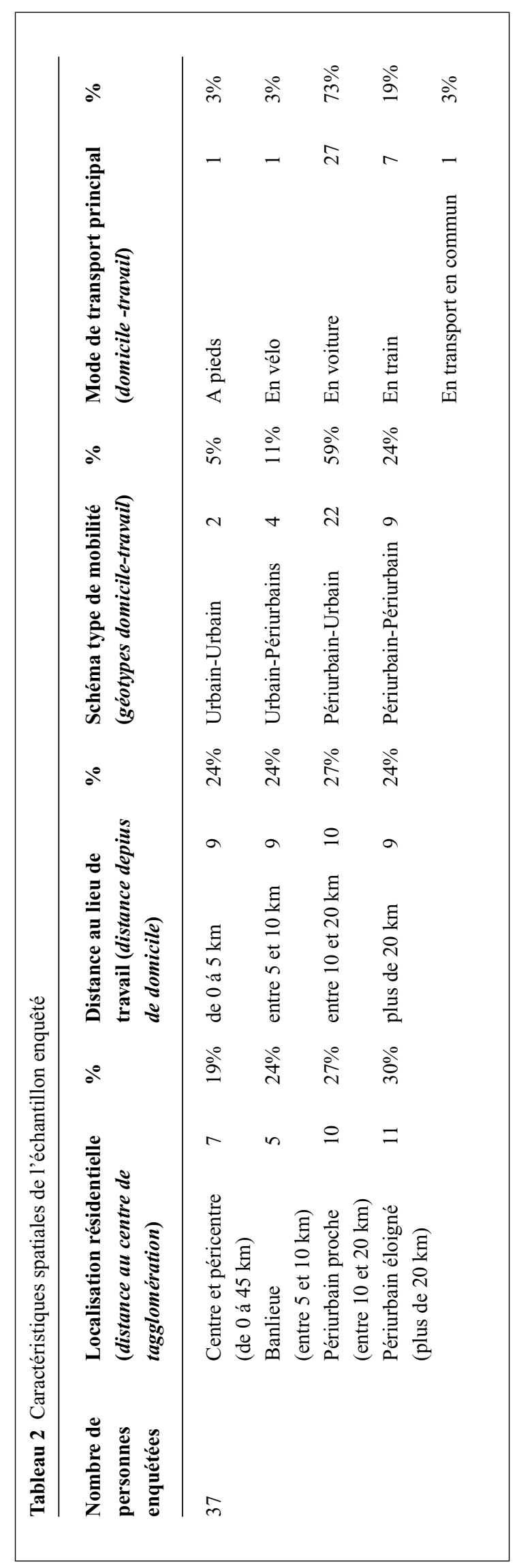

Necplus 


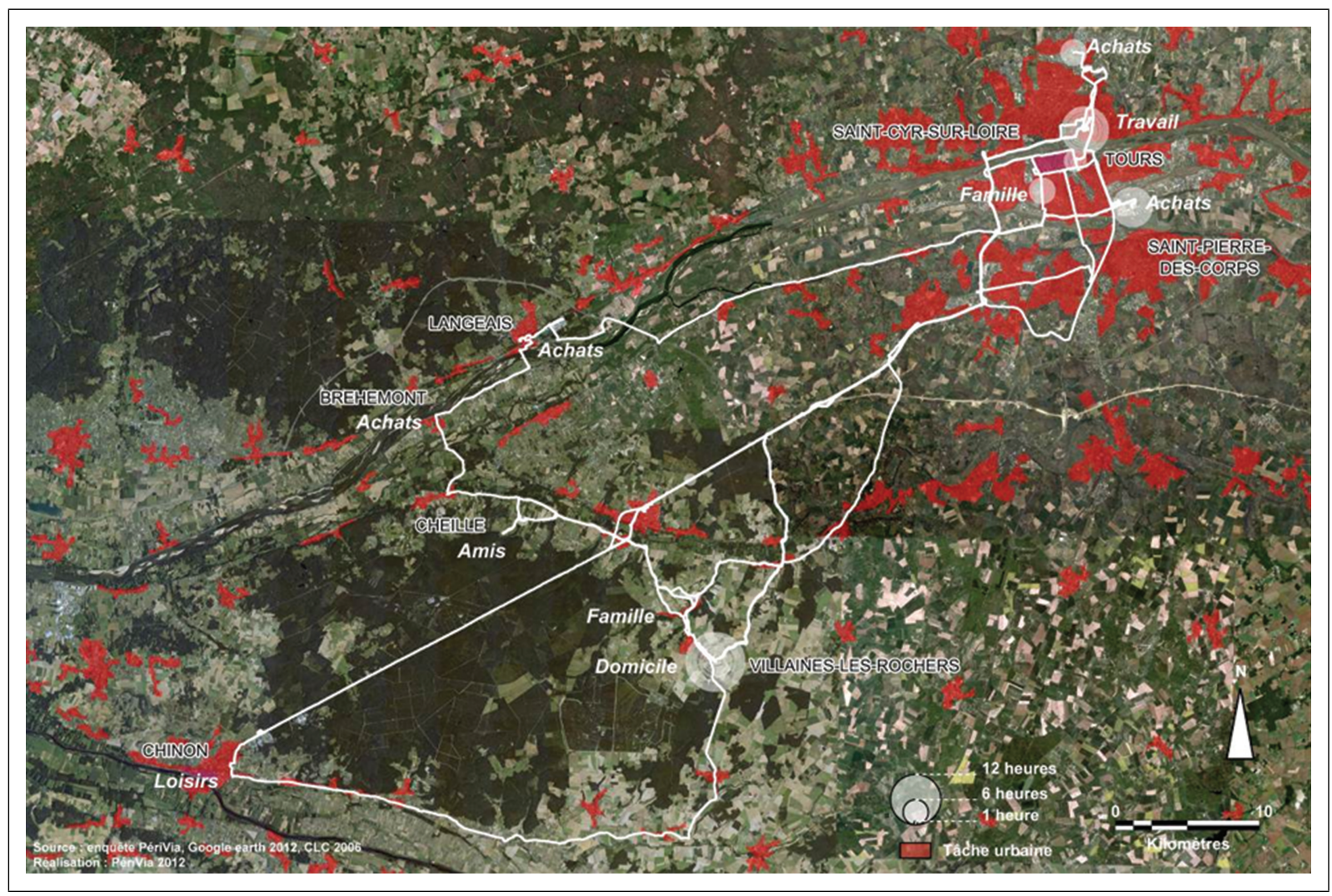

Carte 1 Relevé GPS de J6, sur une semaine type

mobilités », version subjective du sens que les individus accordent à leurs propres mobilités, venant à la suite, compléter ou amender, le relevé objectif des pratiques spatiales quotidiennes. Dans le cadre de ce type d'enquête, le récit de mobilités s'apparente à une variante d'une technique bien connue des sociologues et des géographes, à savoir le « récit de vie ». Celui-ci consiste simplement à recueillir la parole d'un individu à qui on demande, schématiquement, de raconter sa vie comme un récit au sens littéraire du terme, c'est-à-dire en respectant approximativement une chronologie et en ciblant une thématique donnée, ici en l'occurrence l'histoire de ses mobilités quotidiennes. L'enquêté étant amené à parler des choses en tant que celles-ci lui sont à la fois extérieures et en même temps le concernent, le but du récit de mobilités est de connaitre les conditions dans lesquelles se manifestent ces pratiques mobiles et la variété des sens que, dès lors, les individus leur confèrent. C'est par ces différents moyens, en proposant la confrontation d'une certaine réalité spatiale des pratiques mobilitaires (le relevé GPS ; Carte 1), à la construction idéelle que s'en fait l'individu, que nous avons pu atteindre les représentations, les imaginaires et les mythes associés à la mobilité dans les espaces périurbains.

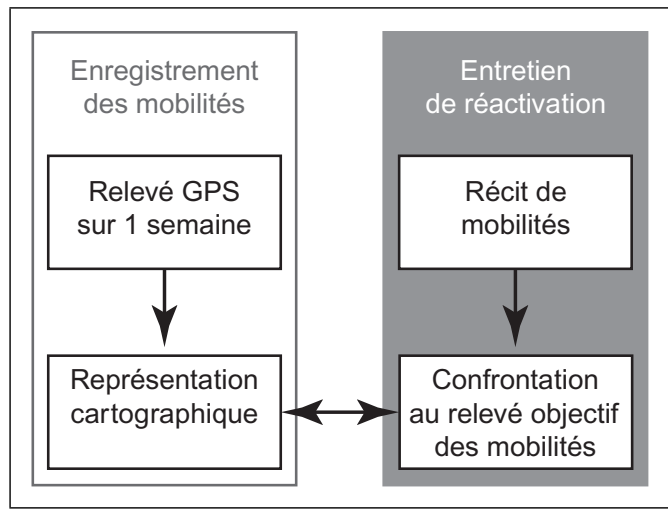

Fig. 1 Protocole d'enquête PériVia (2012)

\section{L'imaginaire périurbain en mutations ?}

\subsection{Quelques caractères constitutifs d'un nouvel imaginaire mobilitaire}

Rejoignant un constat désormais établi, les enquêtes conduites ont d'abord confirmé la dépendance des ménages périurbains à la mobilité, en particulier automobile. La vie quotidienne dans les périphéries urbaines, plus précisément 
la vie de famille, ne semble pas possible sans recours à la voiture. En effet, une des caractéristiques de la mobilité périurbaine est qu'elle résulte d'une programmation complexe [39] liée à la gestion coordonnée de la mobilité de l'ensemble des membres du ménage. À ce titre, la voiture est souvent considérée comme une obligation, voire une norme. Même lorsqu'un réseau de transport en commun est présent sur le territoire et plus ou moins aisément accessible, celui-ci n'arrive pas à supplanter l'automobile.

Supprimer la voiture non. Pour moi c'est inenvisageable... à court terme sûr. . à moyen terme je ne vois pas la supprimer. Qu'il y ait une offre ferroviaire ou pas... c'est le cas ici mais ensuite compte tenu des contraintes familiales je ne vois pas vraiment de solution. ${ }^{4}$

L'imaginaire de la mobilité périurbaine se décline encore en grande part sur le mode automobile. Preuve en est, cette mobilité familiale, essentiellement automobile, est associée à des valeurs extrêmement positives. Elle s'apparente à un sacrifice consenti en vue d'atteindre un certain bien-être. Dans ce contexte, la voiture est un outil assurant l'efficacité des déplacements, un gain de temps pour gérer la complexité des pratiques ou profiter pleinement des offres disponibles sur le territoire.

On est près de tout avec nos véhicules personnels, les transports en commun on les utilise pas. Il y en a, mais on ne les utilise pas. Donc moi je me déplace en voiture. ${ }^{5}$

L'automobile, son usage intense et programmé, entre dans la définition du mode d'habiter périurbain. En ce sens, elle est constitutive $\mathrm{du}$ 《 pack périurbain » [5], et l'imaginaire de la mobilité en contexte périurbain se conforme encore à cette image d'une mobilité libératrice et émancipatrice.

Pour autant, la dimension fonctionnelle n'est pas la seule valeur associée au déplacement. La mobilité périurbaine, chez certains enquêtés, constitue une forme d'agrément. En effet, une part non négligeable de l'imaginaire de la mobilité dans les espaces périurbains se rapporte aux paysages, aux itinéraires, aux parcours et aux étapes qui les jalonnent. Ainsi, le déplacement n'est pas simplement un besoin dérivé des activités, mais devient, dans certains cas, une fin en soi, un moment d'échappement, une pause entre les deux pôles principaux que sont le travail et le foyer. Un moment qui, dès lors, n'appartient qu'à soi et se réalise aussi pour soi, indépendamment du mode de transport utilisé.

\footnotetext{
${ }^{4} \mathrm{H} 1$ : homme, 39 ans, profession intellectuelle supérieure, divorcé, 2 enfants, travaille à Tours et habite à $16 \mathrm{~km}$ de Tours, se déplace principalement en voiture.

${ }^{5} \mathrm{~F} 4$ : homme, 32 ans, profession intermédiaire, marié, sans enfants, travaille à Tours et habite à $7 \mathrm{~km}$ de Tours, se déplace principalement en voiture.
}

Maintenant je suis à Amboise, en travaillant à Tours toujours. Alors là j'adore... j'ai passé un cap où il faut que je me ressource un peu, donc faire la route, Tours-Amboise, sur les bords de Loire, je me sens bien. Je quitte la ville, et quand j'arrive à Amboise, j'ai l'impression d'être en vacances, c'est la petite campagne... ${ }^{6}$.

C'est extrêmement plaisant de prendre le car... pour le paysage parce qu'on traverse deux forêts : la forêt d'Azay-leRideau et surtout la forêt de Chinon qui est splendide. C'est vraiment chouette. Le car, j'arrive pas à lire, alors je rêvasse, je me repose et puis au bout d'un moment on commence à connaître les gens. Ça peut être l'occasion de discuter. ${ }^{7}$

Cela étant, on constate certaines transformations à l'œuvre, plus profondes encore. La mobilité individuelle, principalement automobile, est vécue dans certains cas comme un excès qui appelle en contrepartie, dans un souci mêlé d'économie et d'écologie, la mise en œuvre de logiques d'optimisation par regroupement. En effet, il n'est pas rare, en face des discours prononcé sur le mode individuel, de recueillir un discours associant la mobilité au partage. Ce rôle alloué au déplacement automobile est un des enseignements majeurs que nous avons pu tirer des entretiens réalisés.

Sous les effets de l'injonction au développement durable, on constate un affaiblissement de l'imaginaire de la mobilité-ubiquité, de l'individu-mobile tout-puissant, au profit d'un (ré)enchantement, notamment social, des mobilités. En effet, la mobilité importante dans les espaces périurbains n'est pas vécue comme un privilège. Elle est certes le lot de tout un chacun, mais elle pèse également plus ou moins fortement sur les ménages, en particulier économiquement. Pour cela, la mobilité peut être un motif tout à fait important dans l'évolution des pratiques et la transformation des imaginaires associés aux espaces périurbains à commencer par le développement des relations avec le voisinage plus ou moins élargi. C'est le cas notamment dans le cadre de l'accompagnement des enfants à l'école, entre parents qui, soit ont des enfants scolarisés dans les mêmes établissements, soit mettent à profit leur proximité résidentielle. Parce qu'à un moment les individus jugent le coût, temporel, économique, écologique de leurs pratiques spatiales trop élevé, ces habitants des espaces périurbains font la démonstration d'une capacité à faire évoluer leur conception de la mobilité. Si ce co-déplacement familial et amical est facilement réalisable, il constitue en

\footnotetext{
${ }^{6} \mathrm{~S} 3$ : femme, 42 ans, profession intermédiaire, divorcée, 2 enfants, travaille à Tours et habite à $22 \mathrm{~km}$, se déplace principalement en voiture.

${ }^{7} \mathrm{~S} 1$ : femme, 50 ans, profession intermédiaire, divorcée, 2 enfants, habite à Tours et travaille à $40 \mathrm{~km}$ de Tours, se déplace principalement en car et en train.
} 
outre une des formes les plus acceptables de la mobilité durable.

Moi, j'ai commencé quand ma fille aînée était au collège à Vouvray. Les autres [enfants] étaient encore à l'école, mais ils terminaient à la même heure. C'est vrai que moi ça m'arrangeait que quelqu'un puisse me la ramener. Et eux ça les arrangeait que $\mathrm{j}$ 'amène leur fille le matin. Donc je pense que tout le monde y trouve son compte. ${ }^{8}$

On parle beaucoup de covoiturage, mais ça a toujours existé cette forme de solidarité. C'est vrai que pour les enfants, on se regroupe pour les amener à l'école. C'est tout simplement de l'entraide entre voisins. ${ }^{9}$

La mobilité est un outil permettant, sous certaines conditions, durant le déplacement ou autour de ce dernier, le développement de sociabilités en dehors des activités quotidiennes, professionnelles et domestiques. Elle participe au rayonnement des pratiques plus personnelles qui composent un volet important de l'habiter - bien que celles-ci soient généralement considérées comme absentes dans les espaces périurbains. Si, pour certains, le véhicule individuel peut aussi être envisagé comme un moyen de dissocier, entre les membres d'une même sphère familiale, les activités et les réseaux de connaissance, notamment lorsque ceux-ci sont distants du lieu de vie, dans d'autres cas, au contraire, la situation périurbaine et la mobilité qu'elle implique peuvent aussi devenir un outil privilégié de maintien et d'entretien des liens au sein de la famille.

Ici les enfants peuvent circuler facilement et puis en plus, donc ça faut pas le répéter aux enfants, c'est qu'à Fondettes malgré tout t'es en retrait et... tu peux pas tout le temps être parti... parce que quand t'es en centre-ville, $j$ 'imagine mon fils, si on habitait en centre-ville, ben je le verrais moins. ${ }^{10}$

Le convoiement et l'accompagnement des enfants à leurs diverses activités sont aussi un moyen et un moment de partage entre les membres de la famille, limitant l'individualisation des comportements. À l'encontre d'une image répandue et simplificatrice, celle d'une mobilité sans entrave - associée d'ailleurs par ces mêmes habitants principalement aux espaces urbains - la mobilité périurbaine est dès l'enfance assortie d'un principe de modération, de limitation. Les parents apprennent aux enfants que tout déplacement doit être réfléchi, coordonné, mesuré.

${ }^{8} \mathrm{~V} 1$ : femme, 41 ans, employée, mariée, 3 enfants, travaille à Vouvray, habite à Montlouis-sur-Loire, se déplace principalement en voiture.

${ }^{9}$ M2 : femme, 47 ans, profession intermédiaire, 3 enfants, travaille à Tours, habite à Véretz, se déplace principalement en voiture.

${ }^{10} \mathrm{~S} 2$ : femme, 44 ans, profession intellectuelle supérieure, mariée, 3 enfants, travaille à $60 \mathrm{~km}$ de Tours et habite à $9 \mathrm{~km}$ de Tours, se déplace principalement en train.
Autre signe d'une évolution des représentations et des imaginaires attachés à la mobilité dans les espaces périurbains, on observe une distinction marquante entre les différents modes de transport se rapportant à la question du bien-être social notamment. Alors que la voiture peut être vécue, dans certains cas, comme un mode de déplacement contraignant et pour cela perçue négativement, le train permet quant à lui de « faire autre chose » (se détendre, se cultiver, etc.) et d'accueillir de nouvelles sociabilités.

Le train ça m'a permis également pendant ce temps de trajet de faire des pauses, de pouvoir lire, de pouvoir continuer à travailler si besoin, c'est un lieu d'activités et c'est presque un lieu de convivialité, d'intimité [...] C'est un lieu où je peux tout à fait lire, où je peux tout à fait travailler, où je peux échanger avec mes amis de train, parce que du coup je me suis fait des amis dans le train, voire des collègues qui travaillent avec moi... ${ }^{11}$

Et puis la gare, on rencontre des gens, on discute ! C'est marrant, on se met en condition, avant de commencer à travailler ! [...] Mais vous savez justement, le fait d'habiter à Sainte-Maure-de-Touraine, de prendre le train comme je le fais pour aller bosser sur Tours, et bien ça me donne l'occasion de rencontrer des gens que je n'aurais pas rencontré sur Tours. On rentre en contact avec des gens qui sont dans la même situation que vous, qui travaillent dans une grande ville, et qui habitent à l'extérieur. ${ }^{12}$

Le (ré)enchantement social de la mobilité prend sa source dans les opportunités de rencontre, de découverte, de complicité avec d'autres voyageurs, rendues possibles par l'usage de modes de transport collectifs, et qui en atténuent dès lors la dimension contraignante. La contrainte du déplacement - plus long et impliquant la gestion de plusieurs modes de transport : train, bus urbain et marche est également compensée par la possibilité, certains jours, de recourir à un mode de transport individuel. Ici, on constate l'importance, associée à la pratique plurimodale, du contrôle par l'individu de son inscription spatiale et sociale, faisant par là même de la « non captivité » vis-à-vis d'un mode de transport un élément de distinction socio-spatiale fort, constitutif de l'imaginaire mobilitaire périurbain. Le choix $\mathrm{du}$ train est d'autant plus valorisant qu'il est rationnellement consenti, à la suite d'ajustements et d'essais multiples (comme dans le cas de N2 ; Tableau 3). En outre, dans bien des cas, le transport en commun et en particulier le train, se révèle beaucoup plus commode, au regard d'autres

\footnotetext{
${ }^{11}$ M6 : femme, 55 ans, profession intermédiaire, mariée, 2 enfants, travaille à Tours et habite à $24 \mathrm{~km}$ de Tours, se déplace principalement en train.

12 P5 : homme, 45 ans, profession intermédiaire, divorcé, un enfant, travaille à Tours et habite à $31 \mathrm{~km}$ de Tours, se déplace principalement en train.
} 
solutions partagées tel le covoiturage, en ce sens qu'il permet de maintenir une marge de manœuvre individuelle dans le cadre du déplacement et de préserver l'idéal de liberté associé aux espaces périurbains.

C'est pas facile le covoiturage. Il faut vraiment avoir les mêmes horaires. J'ai essayé de regarder, de me renseigner avec des gens que je connais, mais personne n'a les mêmes horaires. Ou alors, il faut vraiment s'y tenir. . Finalement, le train c'est l'idéal. ${ }^{13}$

Cela étant, l'analyse des conditions citées par les personnes interrogées pour la mise en œuvre de stratégies de mobilité plus durables, comme le train, montre aussi que les raisons d'un changement de mode, induisant une réorganisation à la fois des temps mais aussi du mode de vie des individus, doivent être multiples pour devenir véritablement effectives et perdurer (Tableau 3).

L'usage des transports en commun, pour une mobilité plus durable, s'avère néanmoins complexe à mettre en œuvre quotidiennement. Comme le montre l'exemple de l'enquêté $\mathrm{N} 2^{14}$, la justification du report modal (pour le passage de la voiture au train, avec le Transport Express Régional) se rapporte à un ensemble de raisons qui font référence à des justifications diverses : d'ordre strictement technique ou temporel pour certaines, mais également économique et social pour d'autres. En effet, l'aspect économique se révèle tout à fait important, mais celui-ci se trouve être combiné avec une dimension sociale qui constitue une motivation non négligeable pour accepter une solution de transport pourtant souvent largement plus pesante temporellement (par exemple, dans le cas de N2: 3 heures de transport quotidien en TER, au lieu de 2 heures en voiture). Nous avons ainsi pu constater, lors de nos entretiens, ces différentes formes de sociabilité qui nous étaient rapportées notamment à propos de l'usage des transports en commun, illustrant le fait que la mobilité n'occupe pas seulement une fonction purement instrumentale [40]. La mobilité remplit une fonction sociale à part entière dans les espaces périurbains. On observe un (ré)enchantement de la mobilité, à travers le développement de sociabilités de déplacement. Dans ces conditions, le transport en commun devient un véritable vecteur de co-présence et de dynamiques sociales qui prennent forme pendant la mobilité, jusqu'à déborder le strict espace-temps du déplacement. La mobilité devient non seulement l'occasion de faire de nouvelles connaissances, mais elle s'affirme aussi comme un outil de mise en réseau sur un mode affinitaire, catalyseur d'appartenances

\footnotetext{
${ }^{13}$ M4 : femme, 56 ans, employée, mariée, 2 enfants, travaille à Tours et habite à $65 \mathrm{~km}$ de Tours, se déplace principalement en train et en bus urbain.

${ }^{14} \mathrm{~N} 2$ : femme, 60 ans, employée, mariée, sans enfants, travaille à Tours, habite à $45 \mathrm{~km}$ de Tours, se déplace principalement en train.
}

territoriales, dont les géométries sont à la fois variables, ponctuelles, diffuses et réticulaires [26].

Être dans le train, avec d'autres personnes, ça c'est le côté positif. C'est ma pause quelque part. Et puis on a des relations extérieures. Je vois avec $\mathrm{X}$ on fait de la gym ensemble. C'est d'ailleurs comme ça qu'on s'est retrouvées sur le quai de la gare. Maintenant, comme on s'est reconnues, on s'arrange, on s'emmène à la gym à tour de rôle. On passe chez elle et je me change. Même si c'est pas une amie intime. On se retrouve toujours au même endroit dans le train et elle me réserve ma place. $^{15}$

Se dévoile ainsi progressivement une certaine propension des individus à adapter leurs pratiques quotidiennes, mais aussi, à moyen terme, à anticiper ces adaptations, à travers les transformations de l'imaginaire de la mobilité périurbaine. Les pratiques, comme les imaginaires, sont en mutation. Les transformations de l'imaginaire de la mobilité mises au jour à travers l'enquête, sont le signe de ces mutations en cours, même si celles-ci ne se font pas sans résistance. En effet, l'imaginaire de la mobilité demeure avant tout un imaginaire social, lié étroitement à la réalisation de soi et à la recherche d'un certain bien-être.

Je le fais quand même assez souvent maintenant... un peu plus souvent... de prendre le bus mais j'aime profondément l'autonomie qu'apporte la voiture. [...] C'est vrai que pour moi c'est très chouette la voiture. C'est chouette d'être autonome, de m'arrêter si je veux, de repartir quand je veux. ${ }^{16}$

Des résistances par rapport au report modal sont encore bien présentes, même si on peut observer un certain nombre d'exemples de valorisation de la mobilité, dans sa forme collective notamment, s'appuyant sur des principes de solidarité et de convivialité. L'imaginaire de la mobilité périurbaine reste encore peu lié au souci environnemental. Toutefois, on observe également la résurgence, particulièrement saillante dans les discours collectés, de l'importance de la relation personnelle au déplacement, à son itinéraire, aux paysages traversés et, plus généralement, du souci porté par les individus aux modalités de l'inscription territoriale de leur mobilité.

Ce que je trouve quand même pas mal c'est qu'il est assez diversifié. Parce que tu as quand même la Loire... et puis là tu as une partie du plateau, c'est agricole en fait. Puis ensuite

\footnotetext{
${ }^{15}$ M4 : femme, 56 and, employée, mariée, 2 enfants, travaille à Tours et habite à $65 \mathrm{~km}$ de Tours, se déplace principalement en train et en bus urbain.

${ }^{16} \mathrm{P} 1$ : femme, 51 ans, employée, divorcée, 2 enfants, travaille à Tours et habite à $8 \mathrm{~km}$ de Tours, se déplace principalement en voiture.
} 


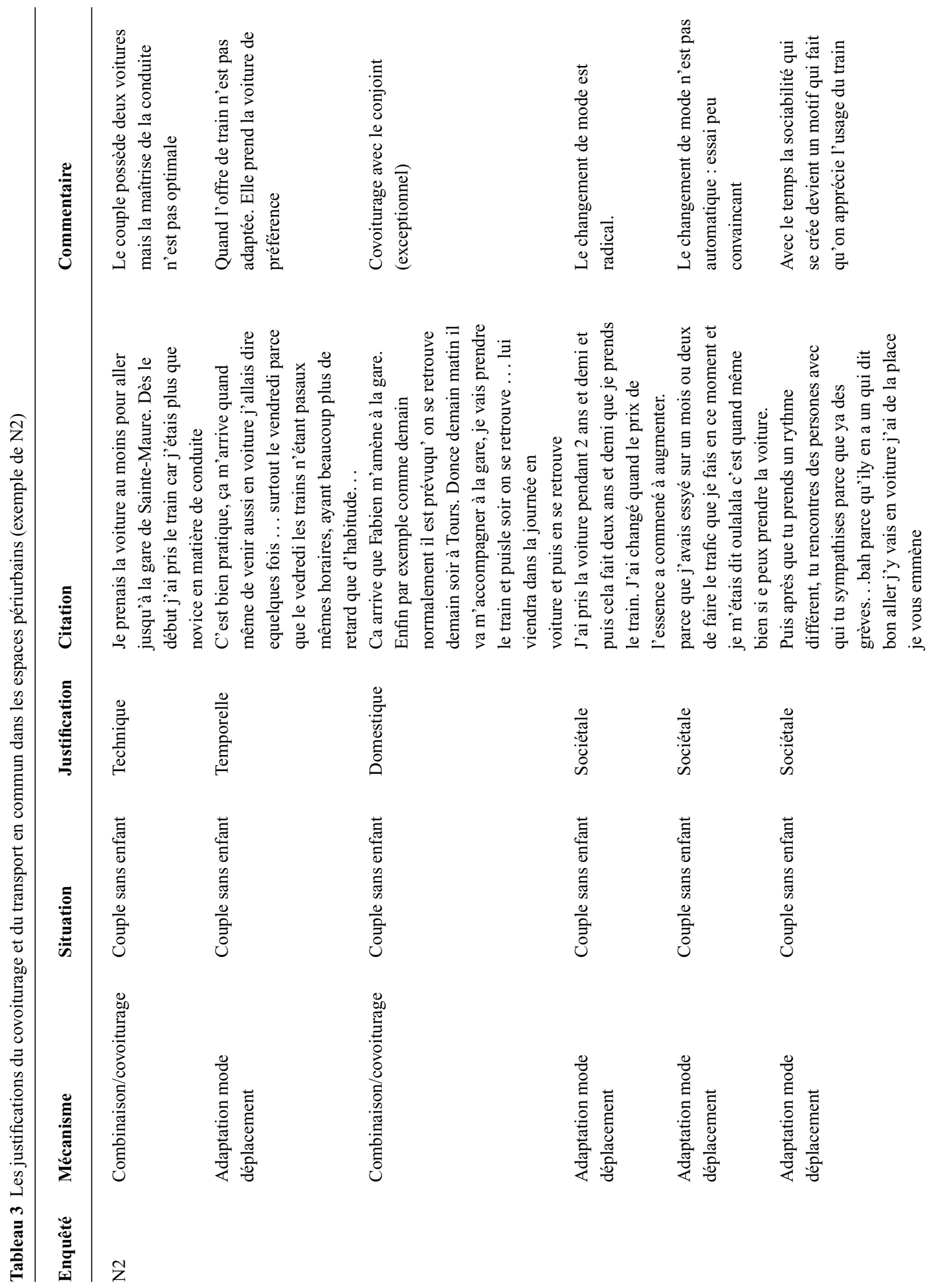

Necplus 
dès que tu traverses... la Loire tu as plus du tout les mêmes paysages. $^{17}$

En ce sens, la nature occupe une place non négligeable, y compris dans l'imaginaire de la mobilité périurbaine, comme élément du système inférant sur les choix des individus en matière de parcours, d'itinéraires, de cheminements. Cependant, contrairement à l'imaginaire associé à l'espace résidentiel, principal réceptacle des valeurs de durabilité, la relation entre nature et mobilité ne demeure encore que rarement envisagée sous l'angle d'une atteinte potentielle à l'environnement susceptible d'enclencher la mise en œuvre d'une plus grande maîtrise des déplacements.

\subsection{Quels imaginaires pour un périurbain durable?}

Si elle ne trouve pas une traduction mécanique dans les pratiques de mobilité et les choix de localisation, l'injonction à la durabilité joue néanmoins un rôle important dans la manière dont les périurbains donnent sens à leur mobilité. La durabilité est bel et bien incorporée comme système de valeurs à l'aune duquel les individus évaluent leurs pratiques. C'est plus particulièrement dans le quotidien que se font jour un certain nombre de concordances possibles entre l'injonction à la durabilité et ses implications dans la structuration des modes d'habiter périurbains.

La mise en pratique des principes du développement durable ressort en premier lieu à travers un discours portant principalement sur un ensemble de «petits gestes », dont le champ d'application privilégié demeure avant tout l'espace résidentiel.

On trie nos déchets, on a du compost ... j'essaie d'acheter des aliments qui ont le moins de déchets possible donc là j'achète plus de barquettes en plastiques [...] Voilà c'est un peu compliqué et le bois c'est très compliqué et en terme de durabilité enfin bref voilà donc bon oui on chauffe relativement peu, on fait attention au chauffage, on utilise au maximum l'énergie du soleil, on met des couvertures pour dormir, on recycle beaucoup de trucs, moi je garde le papier... enfin les enfants ils écrivent sur du papier brouillon, je garde plein de papiers pour faire du bricolage ${ }^{18}$

L'habitat est l'occasion pour les individus, qui se projettent durablement dans un lieu d'ancrage périurbain, de réaliser concrètement l'injonction à la durabilité et de mettre en

\footnotetext{
${ }^{17} \mathrm{H} 1$ : homme, 39 ans, profession intellectuelle supérieure, divorcé, 2 enfants, travaille à Tours et habite à $16 \mathrm{~km}$ de Tours, se déplace principalement en voiture.

${ }^{18} \mathrm{~S} 2$ : femme, 44 ans, profession intellectuelle supérieure, mariée, 3 enfants, travaille à $60 \mathrm{~km}$ de Tours et habite à $9 \mathrm{~km}$ de Tours, se déplace principalement en train.
}

œuvre, pour ce faire, à travers l'investissement dans des dispositifs techniques (efficacité énergétique du bâtiment) et dans des activités (recyclage, production potagère), les changements de leurs comportements.

On a fait changer notre chaudière, enfin la chaudière au fioul existante qui devait avoir 40 ans voir plus donc qui consommait pas mal contre une chaudière à condensation et on a fait mettre deux panneaux solaires pour les sanitaires et l'eau chaude. L'année d'après on a fait changer toutes les fenêtres et porte-fenêtre. . Meilleure isolation, double vitrage. ${ }^{19}$

La durabilité déjà incorporée aux modes de vie périurbains est une durabilité référée au contexte sociétal, c'est-à-dire fortement en lien avec les opportunités proposées ou plébiscitées au niveau collectif. Ainsi les modifications dans l'habitat, relatives aux économies d'énergies à l'isolation ou encore aux éco-gestes, sont largement convoquées par les habitants lorsque ceux-ci se trouvent confrontés à l'impératif de durabilité. La durabilité, jugée acceptable, est celle qui induit une modification technique de l'habitat ou encore des habitudes socialement organisées (ramassage des ordures, codification des produits en fonction de leur consommation énergétique, etc.). L'alimentation est également un domaine auquel la durabilité est reliée, à travers la figure du produit biologique ou des circuits courts (marchés, producteurs locaux).

Cependant, là où les éco-gestes sont jugés comme relativement faciles à mettre en place à l'échelle de l'habitation et de l'alimentation, il faut bien reconnaître que les changements de comportements sont plus difficiles à opérer, car plus complexes, dans le domaine de la mobilité quotidienne.

Oui tout ce qui concerne un petit peu l'ensemble des personnes c'est-à-dire le tri sélectif tout ça voilà... mais les mobilités je ne pense pas... on est attaché à notre grande liberté de mouvement. ${ }^{20}$

La hiérarchie des valeurs associées à la mobilité, elle-même étroitement dépendante des représentations d'un idéal de vie particulièrement bien ancré, constitue un système de référence à propos duquel les individus acceptent difficilement de transiger. Ainsi, l'usage du transport en commun pour H1, père de deux jeunes enfants, paraît inacceptable au nom de l'éducation de ses enfants et de leur bien-être :

\footnotetext{
${ }^{19} \mathrm{~N} 2$ : femme, 60 ans, employée, mariée, sans enfants, travaille à Tours, habite à $45 \mathrm{~km}$ de Tours, se déplace principalement en train.

${ }^{20} \mathrm{~F} 4$ : homme, 32 ans, profession intermédiaire, marié, sans enfants, travaille à Tours et habite à $7 \mathrm{~km}$ de Tours, se déplace principalement en voiture.
} 
Je dirais que dans l'absolu [prendre le train] c'est possible si tu fais abstraction des horaires. C'est-à-dire que si moi j'accepte que mon enfant se lève à $6 \mathrm{~h}$ le matin et. . passe $1 \mathrm{~h} 30 \mathrm{ou} 1 \mathrm{~h}$ à la garderie avant l'école. Si tout est fait pour que la voiture soit supprimée, effectivement dans l'absolu je supprime toutes les activités ou presque du mercredi et du samedi matin. Je leur fais faire plein de garderie et puis elles se débrouillent pour leurs devoirs. Dans ces conditions-là, effectivement la voiture je peux presque la supprimer. . ${ }^{21}$

L'injonction à la durabilité, pour ce qui deviendrait dès lors une mobilité et un périurbain durables, achoppe ici, face à l'imaginaire périurbain, car elle n'est pas suffisamment puissante pour proposer un mode de vie qui préserve les valeurs auxquelles l'individu adhère : l'éducation des enfants, les activités extrascolaires, l'accès à l'emploi, l'ascension sociale, etc. En contrepartie, l'absence ou l'économie de mobilité dans les espaces périurbains n'est pas non plus toujours envisagée comme une situation potentiellement problématique. Les mythes d'une nature et d'un espace résidentiel idéalisés sont pour certains en mesure de se substituer à un potentiel de mobilité et de conférer ainsi sa consistance à l'imaginaire d'un périurbain durable.

C'est une maison qui est construite sur $1600 \mathrm{~m}^{2}$ de terrain, donc j'ai une pelouse, il y a un chien... on a pu installer tous les éléments de jeu quand les enfants étaient petits, les réunions de famille se font sous un grand préau... c'est la maisonnée, le lieu de la famille, des amis. Je ne m'imagine pas un dimanche après-midi devoir prendre la voiture pour aller dans un jardin public ou aux bords de Loire parce que je n'ai pas ça chez moi. Or, là je sors, je fais ce que je veux chez moi, je peux passer l'après-midi chez moi sans avoir à bouger. ${ }^{22}$

L'une des questions qui dès lors nous semble devoir s'imposer, pour résoudre en partie l'enjeu de la mise en durabilité des espaces périurbains, est de savoir, au-delà même de la remise en cause des choix de localisation de ces habitants des périphéries urbaines, si, face à l'impératif de durabilité, l'imaginaire attaché à l'espace résidentiel constitue un ressort suffisamment puissant pour amener les individus à remettre en question une dimension essentielle de leur mode d'habiter, la mobilité, pour au final préserver un certain mode de vie. Ou si les mutations observées, les quelques caractères constitutifs d'un nouvel imaginaire mobilitaire, encore émergeant, sont susceptibles de contribuer

${ }^{21} \mathrm{H} 1$ : homme, 39 ans, profession intellectuelle supérieure, divorcé, 2 enfants, travaille à Tours et habite à $16 \mathrm{~km}$ de Tours, se déplace principalement en voiture.

${ }^{22}$ M6 : femme, 55 ans, profession intermédiaire, mariée, 2 enfants, travaille à Tours et habite à $24 \mathrm{~km}$ de Tours, se déplace principalement en train. de façon suffisamment significative et déterminante à la mise en durabilité des espaces périurbains.

\section{Conclusion}

De plus amples investigations seront nécessaires pour pouvoir répondre à cette question. Cela étant, on peut supposer qu'une voie possible et raisonnable pour la mise en durabilité des espaces périurbains ressortit à la fois de ces deux mouvements, et de la capacité à les envisager comme pouvant être complémentaires et pas simplement opposés. En attendant une réponse plus affirmée, on peut toutefois déjà noter que quelle que soit la dimension abordée, dès que la question de la durabilité est entrée en jeu dans notre enquête, les personnes interrogées ont eu immédiatement recours à un registre de l'ordre de la justification. Preuve s'il en faut que l'injonction à la durabilité et les représentations sociales qui lui sont associées tendent à se diffuser dans les imaginaires périurbains. Une forme de ralentissement semble bel et bien à l'œuvre, façonnant des mobilités davantage partagées, mesurées et communautaires. L'affirmation de la moindre légitimité du mode de vie et du mode d'habiter périurbains, pour des raisons écologiques (consommation d'espaces, automobilité), mais aussi sociales (individualisme, entre soi), est en effet ressentie par les enquêtés, et ce bien que dans les discours elle soit le plus souvent escamotée. Cette injonction à la durabilité est apparue, dans sa globalité, pour ces mêmes enquêtés, comme une norme construite socialement et politiquement à laquelle chacun doit se plier. Cette norme confrontée aux modes de vie favorise la prise de conscience de l'environnement de la mobilité, l'attention pour les espaces. Pour expliquer sa mobilité, c'est-à-dire autant la dimension physique, tangible, du déplacement, que sa dimension idéelle, imaginaire pour partie, l'individu a recours à un ensemble de justifications plus ou moins complexes dont les arguments sont de différents ordres. Dans ce contexte, la durabilité questionne autant la réalité des déplacements, dans un souci d'économie du quotidien, que le discours porté par les individus sur la dimension mobile de leur existence, sur le sens que revêt pour eux le fait d'être en mouvement.

Être mobile signifie faire usage d'un capital pour affirmer ou tendre vers une position spatiale et vers l'affirmation d'une position sociale [41]. Ces positions sont elles-mêmes fortement conditionnées par des imaginaires et un ensemble de représentations associées. Dans les espaces périurbains, l'imaginaire pavillonnaire, synonyme d'un idéal à la fois social et spatial, la famille et la proximité avec la nature, tient toujours une place importante. De son côté, l'imaginaire lié à l'automobile (extension de la maison 
individuelle et moyen d'émancipation) participe également, malgré de fortes contraintes et une inégalité certaine, à maintenir une certaine "idéologie mobilitaire » [42]. Or, depuis l'avènement du paradigme du développement durable, l'imaginaire de la mobilité est en mutation. Tandis que l'injonction à la durabilité semblait condamner d'avance « toute mobilité non durable » et plaidait en faveur d'un regain des valeurs de proximités, d'attachement et d'ancrage, voire d'immobilisme, la mobilité semble se (ré)enchanter. L'analyse conduite dans le cadre de cette recherche a permis de révéler un système plus complexe qu'il n'y paraît, entre la réalité et l'imaginaire des mobilités, entre les pratiques mobilitaires elles-mêmes, entre les conditions de leur réalisation et le sens que les individus leur confèrent. Ainsi, c'est à l'intérieur de ce système, au sein duquel émergent les conditions d'un possible (ré)enchantement, à l'aune des valeurs de sociabilité notamment, qu'il semble désormais falloir penser la conjonction devenue inévitable de deux termes jusqu'alors jugés irrémédiablement antinomiques. En effet, en cherchant à garantir l'ensemble de ses pratiques, l'individu fait la démonstration de sa capacité à transformer sa propre conception de la durabilité, pour l'adapter aux spécificités du mode d'habiter périurbain. $\mathrm{Si}$, dans les discours qui abordent la durabilité à l'œuvre dans les pratiques et modes d'habiter contemporains, la mobilité, malgré son omniprésence en toile de fond demeure souvent un « champ aveugle » de la justification, c'est à travers la mutation de l'imaginaire mobilitaire que paraissent émerger un certain nombre de potentialités, de possibles ressorts, pour une mise en durabilité des espaces périurbains.

L'enquête menée nous a permis d'esquisser quelques traits concernant le rôle des imaginaires dans les espaces périurbains et leur influence sur les représentations associées à la mobilité. En nous appuyant sur ces résultats, nous avons illustré à la fois la singularité, la complexité et les conditions de l'actualisation de chacun des termes d'un système s'articulant autour de trois composants : la mobilité, l'imaginaire et le périurbain. Les constats que nous avons pu dresser, nous conduisent à affirmer que le mode d'habiter périurbain apparaît à la fois comme une liste d'attributs dont chaque individu dépend, la mise en système de ces attributs et le sens que revêt, pour les individus, chacun d'entre eux. Au final, ce qui compte n'est pas le poids accordé à tel attribut ou tel autre du système, par nature d'une grande hétérogénéité. Ce qui importe est la capacité de maintenir à chacun l'accès, par la mobilité notamment, à cette liste d'attributs, ce dans la mesure où la pérennité du système en dépend. L'habiter se définit donc, au-delà des pratiques, par les épreuves qui rendent à chacun ces attributs accessibles ou, à l'inverse, qui menacent leur disponibilité. À l'épreuve de la distance aux différents éléments qui constituent cette liste, s'ajoute, voire prédomine, celle de voir entrer ou sortir certains attributs de la liste elle-même. L'imaginaire de la mobilité est chahuté entre un idéal du tout disponible maintenant et un idéal d'économie quotidienne raisonnable et soutenable.

Dans cette perspective systémique, aménager ne signifie pas (ou plus seulement) transformer volontairement un cadre spatial et temporel établi, ou encore répartir des fonctions au sol. Aménager signifie résoudre les conflits qu'engendrent les difficultés liées au maintien de l'équilibre $\mathrm{du}$ système et rendre à nouveau viables les relations entre chacun des termes qui le définissent, par exemple, en ménageant les potentialités matérielles et idéelles révélées à travers l'étude des imaginaires mobilitaires. Quels rôles peuvent ainsi jouer les questions d'esthétique du déplacement ou encore de socialisation à travers la mobilité dans le ménagement des espaces périurbains ? Guidés par des représentations majoritairement négatives du périurbain, et de la mobilité qui lui est associée, les acteurs politiques peinent à prendre en compte ces mutations à l'œuvre et, en particulier, à intégrer l'imaginaire mobilitaire renouvelé comme un levier potentiel pour la réalisation d'un périurbain durable. La durabilité périurbaine repose aussi sur la capacité (ou l'incapacité) des acteurs à penser ces espaces comme pouvant être durables. Un basculement vers un mode de pensée plus enclin à intégrer la complexité du système ouvrirait une place plus large à la recherche des conditions - parmi lesquelles les multiples dimensions d'un imaginaire mobilitaire en mutation - propices à l'émergence des conditions d'une habitabilité périurbaine compatible avec les enjeux de durabilité.

\section{Bibliographie}

1. Debarbieux B (1995) Imagination et imaginaire géographiques. In: Bailly A et al. (éds.) Encyclopédie de géographie. Économica, pp 875-888.

2. Desnoilles R et al. (2012) L'imaginaire géographique, un contrepoint à la réalité ? Perspectives, pratiques et devenirs. In: Bédard $\mathrm{M}$ et al. (éds.) L'imaginaire géographique. Perspectives, pratiques et devenirs. Presses de l'Université du Québec, pp 1-17.

3. Raymond $\mathrm{H}$ et al. ([1966] 2001) L'habitat pavillonnaire. L'Harmattan.

4. Mercier G (2006) La norme pavillonnaire : mythologie contemporaine, idéal urbain, pacte social, ordre industriel, moralité capitaliste et idéalisme démocratique. Cahiers de géographie $d u$ Québec 50 : 207-239.

5. Cailly L (2008) Existe-t-il un mode d'habiter spécifiquement périurbain? EspacesTemps.net, http://test.espacestemps.net/articles/ existe-t-il-un-mode-drsquohabiter-specifiquement-periurbain/

6. Sénécal G (1992) Aspects de l'imaginaire spatial : identité ou fin des territoires? Annales de Géographie $563: 28-42$.

7. Barrère A, Martuccelli D (2005) La modernité et l'imaginaire de la mobilité : l'inflexion contemporaine. Cahiers internationaux de sociologie $118: 55-79$.

8. Moscovici S (1984) Psychologie sociale. PUF.

9. Laffont GH et al. (2013) L'espace du nouveau monde: mythes et ancrages territoriaux. PUR. 
10. Urry J ([2000] 2005) Sociologie des mobilités. Une nouvelle frontière pour la sociologie? Armand Colin.

11. Wiel M (1999) La transition urbaine. Mardaga.

12. Dodier R (2007) Les pratiques spatiales des périurbains. In: Devisme L (éds.) La structuration du périurbain. Synthèse bibliographique. PUCA, DRE, CETE, MSH Ange Guépin, pp 41-43.

13. Piolle X (1990) Mobilité, identités, territoires. Revue de géographie de Lyon 65 : 149-154.

14. Allemand S et al. (2004) Les sens du mouvement. Modernité et mobilité dans les sociétés urbaines contemporaines. Belin.

15. Bailleul H, Feildel B (2011) Le sens des mobilités à l'épreuve des identités spatiales : un éclairage par le récit de vie spatialisé et l'herméneutique cartographique. In: Depeau S, Ramadier T (éds.) Se déplacer pour se situer. Place en jeu, enjeux de classes, PUR, pp 25-55.

16. Vincent S (2008) Les « altermobilités 》: analyse sociologique d'usages de déplacements alternatifs à la voiture individuelle. Des pratiques en émergence ? Thèse de doctorat en sociologie, Université Paris 5-René Descartes, Paris.

17. Fol S (2009) La mobilité des pauvres. Pratiques habitantes et politiques publiques. Belin.

18. Gerber P, Carpentier S (2013) Mobilités et modes de vie, vers une recomposition de l'habiter. PUR.

19. Périvia (2012), Le périurbain à l'épreuve des modèles d'habiter. La viabilité périurbaine entre théorie(s) et pratique(s). Rapport de recherche PUCA, MEDD, Université François Rabelais, Tours.

20. Berque A et al. (2006) La ville insoutenable. Belin.

21. Morel-Brochet A (2007) À la recherche des spécificités du mode d'habiter périurbain dans les représentations et les sensibilités habitantes. Norois $205: 23-36$.

22. Goyon M, Ortar N (2009) Désir de maison à l'aune du parcours résidentiel. Articulo - Journal of Urban Research, http://articulo.revues.org/1427

23. Légué $P$ (2000) La maison individuelle, un idéal de vie. Informations sociales $130: 28-36$.

24. Billard G, Brennetot A (2009) Le périurbain a-t-il mauvaise presse ? Analyse géoéthique du discours médiatique à propos de l'espace périurbain en France. Articulo - journal of Urban Research, http://articulo.revues.org/1372

25. Vanier M (2012) Dans l'épaisseur du périurbain. Espaces et sociétés 148-149:211-218.
26. Terrhabmobile (2013) Lorsque la mobilité territorialise. Des pratiques individuelles de mobilité à la mobilisation de solidarités collectives. EspaceTemps.net, http://www.espacestemps. net/articles/lorsque-la-mobilite-territorialise/

27. Abbas Y (2008) Environnements néo-nomades, système écologique ? In: Dervin F, Ljalikova A (éds.) Regards sur les mondes hypermobiles. L'Harmattan, pp 19-36.

28. Tricoire F (2013) Le pouvoir des mythes dans la société française postmoderne. Des créatifs au social, du social au mythe.M@gm@ $11: 1-7$.

29. Marzloff B (2005) Mobilités, trajectoires fluides. CERTU, Éditions de l'Aube, $240 \mathrm{p}$.

30. Fourny M-C, Lajarge R (2011) Au-delà de la banlieue, c'est encore la périphérie. Les espaces périurbains au prisme de la norme aménagiste, Les banlieues ou la périphérie en tant que laboratoire de l'emprise de la norme, Grenoble.

31. Ascher F (2001) Les nouveaux principes de l'urbanisme. Éditions de l'Aube.

32. Lévy J (2010) Le développement urbain durable entre consensus et controverse. L'information géographique $74: 39-50$.

33. Newman P, Kenworthy J (1989) Cities and automobile dependance: an internationnal sourcebook. Aldershot.

34. Orfeuil J-P, Soleyret D (2002) Quelles interactions entre les marchés de la mobilité à courte et longue distance ? Recherche Transports Sécurité 76 : 208-221.

35. Feildel B, Martouzet D (2012) La mobilité comme modalité de l'ancrage : enrichir l'évaluation de la durabilité des espaces périurbains. Recherche Transports Sécurité 28 : 271-289.

36. Stock M (2004) L'habiter comme pratique des lieux géographiques. EspaceTemps.net, http://www.espacestemps.net/ articles/Irsquohabiter-comme-pratique-des-lieux-geographiques/

37. Martouzet $\mathrm{D}$ et al. (2010) La carte : fonctionnalité transitionnelle et dépassement du récit de vie, Natures Sciences Sociétés 18(2) : $158-170$

38. Chalas Y (2000) L'invention de la ville. Anthropos.

39. David O (2013), Les équations temporelles et spatiales des familles périurbaines, EspacesTemps.net, Vivre le périurbain, http://www.espacestemps.net/articles/les-equationstemporelles-et-spatiales-des-familles-periurbaines/, 22/5/2013.

40. Cresswell T (2006) On the move: Mobility in the modern western world. Routledge.

41. Lussault M (2007) L'homme spatial. Seuil.

42. Mincke C, Montulet B (2010) L'idéologie mobilitaire. Politique, revue de débats 64, La nouvelle obsession de la mobilité, http://www.politique.eu.org/spip.php?article1075, 25 mai 2011. 